\title{
Preventing aggressive/violent behavior: a role for biomarkers?
}

\author{
Martina Pinna ${ }^{1,2}$ \& Mirko Manchia ${ }^{*}, 3,4$ \\ ${ }^{1}$ Unit of Psychiatry, Department of Mental Health \& Addiction Services, Regional Health Agency, Oristano, Italy \\ ${ }^{2}$ Section of Neurosciences \& Clinical Pharmacology, Department of Biomedical Sciences, University of Cagliari, Cagliari, Italy \\ ${ }^{3}$ Department of Pharmacology, Dalhousie University, Halifax, Nova Scotia, Canada \\ ${ }^{4}$ Section of Psychiatry, Department of Medical Sciences \& Public Health, University of Cagliari, Cagliari, Italy \\ * Author for correspondence: mirkomanchia@unica.it; mirko.manchia@dal.ca
}

First draft submitted: 22 April 2017; Accepted for publication: 11 May 2017; Published online: 11 September 2017

Keywords: aggression $\bullet$ biomarkers $\bullet$ personalized medicine $\bullet$ pharmacogenomics $\bullet$ prognostic models $\bullet$ severe mental illness

\section{The burden of aggressive/violent behavior}

Despite a declining rate in the past 10 years, aggressive/violent behavior remains among the main causes of worldwide premature mortality [1]. Associated direct (medical and nonmedical expenditure) and indirect (longterm societal implications) socio-economic costs are also substantial $[2,3]$. These have been estimated in 2001 at approximately $5 \%$ of the gross national product of industrialized countries, and as much as $14 \%$ of the gross national product of low-income countries [3].

In this context, policy makers have attempted to enact interventions that could prevent the development of aggression and violence in at-risk populations (i.e., abused children and troubled youth, alcohol and substance abusers, prisoners). These public health approaches that include, among the others, education, behavior change, policy, engineering and environmental support, have proven to be effective in reducing the rates of aggression/violence as well as its associated economic costs [4].

Nonetheless, there is still a substantial proportion of individuals who enact aggressive/violent behavior over the lifetime course that is not targeted by these interventions. Further, such interventions are implemented only once aggressive behavior has already manifested itself.

By definition, primary preventive strategies intend to avoid the manifestation of a specific disease or event before it occurs. To be effective, preventive approaches need to identify risk factors that can determine the onset of an illness or of an event in an exposed population.

In the case of aggressive/violent behavior, there is a multitude of determinants (clinical, environmental and biological) that set the liability threshold for its manifestation. It is plausible that predictive models integrating different levels of information (from phenotypic to 'omics' data) might help identifying with higher accuracy individuals at risk for aggression/violence compared with those relying only, for instance, on clinical data. However, the inclusion of biological markers (henceforth biomarkers) in such models can be achieved only if they are identified in longitudinal prospective studies and are replicated in independent samples. Importantly, this process could also lead to a more precise understanding of the neurobiological underpinnings of aggression/violence.

Here, we critically discuss the recent advancements in the identification of biological markers of aggression and the eventual barriers toward their implementation in predictive models. To this end, we will first briefly present the current knowledge on the neurobiological make up of aggression/violent behavior. Next, we will discuss how the phenotypic heterogeneity of aggression might have hindered the identification of reliable biomarkers. Finally, we will present data on available biological markers of aggression that might be suitable for implementation in predictive models. 


\section{Neurobiological basis of aggressive/violent behavior: insights for preventive strategies?}

Aggressive behavior appears to have specific neurocircuitry and neurochemical underpinnings [5]. Indeed, several structures of the central nervous system (CNS) appear to regulate those emotional and behavioral components, that, when altered, can precipitate aggression: the amygdala and the limbic prefrontal cortex (PFC), including the orbitofrontal cortex (OFC) and the anterior cingulate cortex [5,6]. Specifically, decreased amygdala volumes have consistently been found in aggressive individuals [5,7]. In a large longitudinal study, Pardini et al. found that adult men with lower amygdala volume were at increased risk for exhibiting future aggression, violence and psychopathic traits, even after controlling for earlier levels of these features and several potential confounds [7]. Concerning the PFC, there is evidence that smaller volumes of the OFC and of the anterior cingulate cortex are associated with increased aggression [5]. Of interest, Cristofori et al. observed that Vietnam War veterans who suffered penetrating brain lesions at the level of the dorsolateral PFC had a more positive implicit attitude toward violence compared with healthy controls [8].

These structural modifications associated to aggressive/violent behavior appear to translate in specific patterns of altered connectivity among these brain areas [5]. Studies with functional Magnetic Resonance Imaging (MRI) has shown that amygdala responsivity appears to be increased in aggressive individuals [9-11]. Indeed, compared with controls, unmedicated individuals with intermittent explosive disorder showed greater amygdala response to angry (vs neutral) facial expressions [10]. Further, amygdala activation to angry faces was correlated with number of prior aggressive acts [10]. And an increased activity of the left amygdala was also observed in male borderline personality patients during anger induction and aggression phases [11].

Another functional alteration in brain connectivity observed in aggressive individuals is the decreased coupling between amygdala and PFC, particularly OFC [9]. Specifically, Coccaro et al. found that, besides amygdala hyperresponsivity, individuals with intermittent explosive disorder did not show amygdala-OFC connectivity during responses to angry faces [9].

Taken together, these findings highlight specific structural and functional alterations in the brain of aggressive individuals. The identification of associated brain signatures, such as decreased amygdala volume, anteceding the manifestation of aggression, make these brain regions plausible trait biomarkers.

Specific neurochemical alterations have also been associated with aggression [12]. Among the various neurochemical pathways putatively involved, the serotonin (5-hydoxytryptamine [5-HT]) system has been the focus of extensive investigations [13,14]. A parsimonious mechanistic neurobiological model linking the 5-HT system with aggression points to behavioral inhibition as a key mediator [14]. According to this model, a greater activity of the 5-HT system would correspond to a greater behavioral inhibition of the aggressive individual when confronted with threat or frustration [14]. Thus, several elements of the serotonergic system, such as 5-HT peripheral or cerebrospinal fluid levels, 5-HT receptors, metabolic enzymes and their encoding genes, have been tested as plausible biomarkers of aggression [13].

However, the identification of validated biomarkers of aggression among components of the 5-HT system remains in a quandary. Of note, this gloomy scenario applies also to biomarkers pertaining to neurochemical and metabolic pathways other than 5-HT one [15]. One possible explanation for the absence of validated biomarkers of aggression lies in the substantial heterogeneity of clinical (phenotypic) trait and in its multifactorial nature. These aspects are discussed in the following sections.

\section{The multifactorial nature of aggressive/violent behavior: a barrier to the identification of reliable biomarkers?}

Aggressive/violent behavior is heterogeneous in its phenotypic manifestation as well as in its origin. From a phenomenological point of view, aggression can be classified as impulsive or premeditated/instrumental [14,16]. Typically, impulsive aggression is enacted in response to a threat or a provocation, while premeditated/instrumental aggressions are committed with the purpose of gaining something [16]. Both types of aggression could be also pathological, in other words, committed in the context of a severe mental illness (for instance, psychotic patients might act on delusions or hallucinatory command, manic bipolar patients can present impulsive aggression in the context of behavioral disinhibition). This dichotomous classification is not exhaustive as aggression has a multifaceted clinical expression, but represents a relatively solid operational framework for neurobiological research.

The pathogenesis of aggressive/violent behavior is also heterogeneous [17]. Twin studies point to a substantial heritability of this trait [18]. Consequently, a recent molecular genetic study using a hypothesis-free genome-wide approach, has recently identified markers on chromosome $2 \mathrm{p} 2$ associated with aggressive behavior in children [19]. 
Further, these authors were able to confirm that a substantial proportion (10-54\%) of phenotypic variation was explained by common genetic variants [19].

At the same time, as a substantial proportion of heritability (up to 50\%) remains unexplained, it is conceivable that environmental factors might play a role in modulating the liability threshold toward aggression [20]. These environmental moderators might act on the genetic liability threshold during the developmental trajectory from childhood to adulthood or even before birth [21]. In keeping with this hypothesis, Wang et al. (2012) found that the methylation of the 5-HT transporter was altered in peripheral white blood cells of individuals who were physically aggressive during childhood [22].

However, the advancement in the understanding of the genetic and epigenetic make up of aggression has not yet translated into validated panels of biomarkers. As mentioned previously, this transition could have been hindered by the inherent phenotypic heterogeneity of aggression and by the lack of uniformity in the methodology used for the assessment of this trait across studies (i.e., retrospective self-reported information vs prospective data collection based on structured assessment tools). One obvious solution, although not always practical, for overcoming the issue of phenotypic heterogeneity is to enlarge the sample size under study. However, this increase should be substantial, as phenotypic heterogeneity of $50 \%$ raises the required sample size to achieve $90 \%$ of statistical power by approximately three-times in genetic studies [23].

\section{Biomarkers of aggressive/violent behavior: a role for metabolomics?}

Reliable biomarkers of aggressive/violent behavior might be identified by taking advantage of molecular approaches that allow the detection of biochemical signatures associated with distinct biological make ups. Metabolomics permits indeed the extensive profiling of sets of small molecules within cells, tissues and biofluids [24]. At least two characteristics make metabolomics suitable for the analysis of a complex, heterogeneous trait such as aggressive/violent behavior. First, changes in metabolome are amplified relative to changes in gene expression (transcriptome) and the proteome, facilitating the identification of distinct biochemical signatures that could be tested as biomarkers. Second, compared with transcriptomics and proteomics, metabolomics is less context dependent, in other words, is less influenced by changing physiological, pathological or developmental states of the cell [24,25].

Thus, it is not surprising to observe a growing interest in the use of metabolomics in aggressive/violent behavior. In fact, the research consortium ACTION (Aggression in Children: Unraveling gene-environment interplay to inform Treatment and InterventiON strategies) funded by the EU is collecting biological samples from large comprehensive longitudinal cohorts to identify panels of risk biomarkers for aggression. Beside metabolomics, this approach will allow collection of genomic and epigenomic data. This approach should disentangle the heterogeneity of aggression and highlight pathways from molecule to phenotype.

\section{Conclusion \& future perspective}

In summary, aggressive/violent behavior is a complex heterogeneous trait causing a substantial public health burden. Preventive strategies have been focused so far on public health and societal approaches, but have not relied on biological information given the lack of validated biomarkers. We have highlighted sets of biomarkers, such as those identified through neuroimaging, which might be tested in clinical settings, while other areas of research (genomic, epigenomics and metabolomics) have not yet produced validated findings. It is conceivable that in the near future large collaborative research efforts will identify panels of 'omics' biomarkers that might identify with adequate sensitivity and specificity individuals at risk for aggressive/violent behavior.

Financial \& competing interests disclosure

The authors have no relevant affiliations or financial involvement with any organization or entity with a financial interest in or financial conflict with the subject matter or materials discussed in the manuscript. This includes employment, consultancies, honoraria, stock ownership or options, expert testimony, grants or patents received or pending, or royalties.

No writing assistance was utilized in the production of this manuscript.

\section{References}

1. GBD 2015 Mortality and Causes of Death Collaborators. Global, regional, and national life expectancy, all-cause mortality, and cause-specific mortality for 249 causes of death, 1980-2015. A systematic analysis for the Global Burden of Disease Study 2015. Lancet 388(10053), 1459-1544 (2016). 
2. Waters H, Hyder A, Rajkotia Y, Basu S, Rehwinkel JA, Butchart A. The economic dimensions of interpersonal violence. In: Department of Injuries and Violence Prevention (Eds). WHO, Geneva, Switzerland (2004).

3. Pfizer. Responding to the global public health challenge of violence. The Pfizer Journal, Global Edition. 11(1) (2001).

4. Haegerich TM, Dahlberg LL, Simon TR et al. Prevention of injury and violence in the USA. Lancet 384(9937), 64-74 (2014).

5. Rosell DR, Siever LJ. The neurobiology of aggression and violence. CNS Spectr. 20(3), 254-279 (2015).

6. Hoptman MJ. Impulsivity and aggression in schizophrenia: a neural circuitry perspective with implications for treatment. CNS Spectr. 20(3), 280-286 (2015).

7. Pardini DA, Raine A, Erickson K, Loeber R. Lower amygdala volume in men is associated with childhood aggression, early psychopathic traits, and future violence. Biol. Psychiatry 75(1), 73-80 (2014).

8. Cristofori I, Zhong W, Mandoske V et al. Brain regions influencing implicit violent attitudes: a lesion-mapping study. J. Neurosci. 36(9), 2757-2768 (2016).

9. Coccaro EF, McCloskey MS, Fitzgerald DA, Phan KL. Amygdala and orbitofrontal reactivity to social threat in individuals with impulsive aggression. Biol. Psychiatry 62(2), 168-178 (2007).

10. McCloskey MS, Phan KL, Angstadt M, Fettich KC, Keedy S, Coccaro EF. Amygdala hyperactivation to angry faces in intermittent explosive disorder. J. Psychiatr. Res. 79, 34-41 (2016).

11. Herpertz SC, Nagy K, Ueltzhoffer K et al. Brain mechanisms underlying reactive aggression in borderline personality disorder-sex matters. Biol. Psychiatry doi:10.1016/j.biopsych.2017.02.1175 (2017) (Epub ahead of print).

12. Yanowitch R, Coccaro EF. The neurochemistry of human aggression. Adv. Genet. 75, 151-169 (2011).

13. Manchia M, Carpiniello B, Valtorta F, Comai S. Serotonin dysfunction, aggressive behavior, and mental illness: exploring the link using a dimensional approach. ACS Chem. Neurosci. 8(5), 961-972 (2017).

14. Coccaro EF, Fanning JR, Phan KL, Lee R. Serotonin and impulsive aggression. CNS Spectr. 20(3), 295-302 (2015).

15. Hagenbeek FA, Kluft C, Hankemeier T et al. Discovery of biochemical biomarkers for aggression. a role for metabolomics in psychiatry. Am. J. Med. Genet. B Neuropsychiatr. Genet. 171(5), 719-732 (2016).

16. Volavka J. The neurobiology of violence: an update. J. Neuropsychiatry Clin. Neurosci. 11(3), 307-314 (1999).

17. Manchia M, Fanos V. Targeting aggression in severe mental illness: the predictive role of genetic, epigenetic, and metabolomic markers. Prog. Neuropsychopharmacol. Biol. Psychiatry 77, 32-41 (2017).

18. Porsch RM, Middeldorp CM, Cherny SS et al. Longitudinal heritability of childhood aggression. Am. J. Med. Genet. B Neuropsychiatr. Genet. 171(5), 697-707 (2016).

19. Pappa I, St Pourcain B, Benke K et al. A genome-wide approach to children's aggressive behavior. The EAGLE consortium. Am. J. Med. Genet. B Neuropsychiatr. Genet. 171(5), 562-572 (2016).

20. Tremblay RE, Nagin DS, Seguin JR et al. Physical aggression during early childhood: trajectories and predictors. Pediatrics 114(1), e43-e50 (2004).

21. Tremblay RE, Szyf M. Developmental origins of chronic physical aggression and epigenetics. Epigenomics 2(4), 495-499 (2010).

22. Wang D, Szyf M, Benkelfat C et al. Peripheral SLC6A4 DNA methylation is associated with in vivo measures of human brain serotonin synthesis and childhood physical aggression. PLoS ONE 7(6), e39501 (2012).

23. Manchia M, Cullis J, Turecki G, Rouleau GA, Uher R, Alda M. The impact of phenotypic and genetic heterogeneity on results of genome wide association studies of complex diseases. PLoS ONE 8(10), e76295 (2013).

24. Kell DB, Oliver SG. The metabolome 18 years on: a concept comes of age. Metabolomics 12(9), 148 (2016).

25. Kell DB. Metabolomic biomarkers: search, discovery and validation. Expert Rev. Mol. Diagn. 7(4), 329-333 (2007). 